\title{
DUALISMO ECONOMICO E MOBILITÀ SOCIALE IN ITALIA. UN CONFRONTO TRA CENTRO-SETTENTRIONALI, MERIDIONALI E IMMIGRATI INTERNI
}

\author{
Nazareno Panichella \\ Dipartimento di Scienze Sociali e Politiche \\ Università degli studi di Milano \\ nazareno.panichella@unimi.it
}

Discussion paper

This paper studies the association between south-to-north internal geographical mobility and social mobility in Italy. Two issued are analysed: the association between migration and social mobility pathways, comparing the movers with the stayers, i.e. those who did not experienced any episode of geographical mobility; b) whether the effect of south-tonorth mobility changes according to gender and education.

Analyses are based on the Longitudinal Survey on Italian Households (ILHS) and use Mobility tables and Linear Regression Panel Models with random effects. Results shown that the social mobility of internal migrants are characterized by three main pathways: a) to the urban working class, which concerned the southerners originally from the lower classes, especially the children of peasants and laborers; $b$ ) to the white collars, which instead mainly concerned the bourgeois and the white-collar middle class; c) mixed pathways, which involved people from the petty bourgeoisie and the urban working class. Results also shown a gender divide, where a positive effect of geographical mobility is found for men and a negative one for women. Finally, the negative effect among women is confirmed only when they are lower educated.

Keywords: dualismo territoriale, migrazioni interne, disuguaglianze, mobilità sociale. 


\section{Introduzione}

Gli studi di stratificazione sociale hanno spesso paragonato la società a una scala, sui gradini della quale si muovono gli individui che ne fanno parte. Le ricerche empiriche si sono così concentrate sullo studio della mobilità verticale, ovvero sul passaggio tra posizioni sociali che, appunto, analogamente ai gradini della scala, possono essere ordinati gerarchicamente all'interno della struttura occupazionale (Cobalti e Schizzerotto 1994). Anche il classico studio di Sorokin (1927) ha definito il fenomeno con una metafora spaziale, dove i canali di mobilità sociale sono stati definiti come "membrane poste tra gli individui", o anche come "buchi", "scale" o "ascensori". Secondo Sorokin, d'altra parte, gli individui si spostano non solo verticalmente, ma anche lungo molteplici dimensioni corrispondenti ai diversi fattori di differenziazione delle posizioni sociali. Dunque, accanto alla mobilità verticale la ricerca di Sorokin ha considerato anche forme di mobilità di tipo orizzontale, di cui l'esempio più noto e rilevante è quello della mobilità geografica ${ }^{1}$. Anche le ricerche di Lipset e Bendix (1959) e di Blau e Duncan (1967) hanno preso in seria considerazione la mobilità geografica, e in particolar modo gli spostamenti dalle aree rurali a quelle più ricche $\mathrm{e}$ industrializzate. Per questi studi, la migrazione, sia interna che internazionale, rappresenta un meccanismo sociale che ridefinisce la distribuzione della forza lavoro in diverse aree geografiche e, quindi, influenza le opportunità di mobilità sociale degli individui che si spostano da una struttura occupazionale a un'altra.

Le ricerche empiriche sulla mobilità sociale in Italia hanno trascurato il ruolo della mobilità geografica, concentrandosi soprattutto sulla misurazione empirica del fenomeno e sull'analisi dell'andamento nel tempo della mobilità sociale (Paci 1982; Cobalti 1995). Nel nostro paese, però, gli spostamenti territoriali interni fanno parte di un fenomeno di lunghissimo periodo che ha caratterizzato la storia della penisola italiana in maniera particolare rispetto al resto d'Europa. In Italia ci sono stati importanti migrazioni interne dalle montagne verso le pianure e le città, dalle regioni del Nord-est e dal Centro verso quelle del Nord-ovest e dal Mezzogiorno verso il Centro-nord (Ballarino e Panichella, 2021).

Questo studio si concentra sulla migrazione interna più importante della recente storia del nostro paese: quella dal Sud verso le regioni del Centro-nord. L'obiettivo è studiare la mobilità sociale in Italia da un punto di vista "orizzontale", vale a dire confrontando i percorsi occupazionali dei meridionali che si sono trasferiti nel Centronord con quelli dei centro-settentrionali e dei meridionali che non sono emigrati. Le differenze dei percorsi di mobilità del Centro-nord e del Sud sono state oggetto di altri studi (si veda il par. 2), e le persistenti situazioni di svantaggio delle regioni meridionali rispetto al resto del paese sono piuttosto note. Tuttavia, a differenza della letteratura sul

\footnotetext{
${ }^{1}$ Le altre forme di mobilità orizzontale sono: la circolazione intra-professionale degli individui, la circolazione inter-familiare, lo spostamento di cittadinanza tra individui, la circolazione inter-religiosa e quella inter-politica (Sorokin 1927).
} 
tema, questo lavoro considera anche la dimensione territoriale della disuguaglianza sociale, comparando la mobilità sociale degli emigrati interna con quella dei centrosettentrionali e dei meridionali non emigrati, considerando, inoltre, anche le differenze di genere e di istruzione. Quali sono stati i percorsi di mobilità sociale intergenerazionale degli immigrati interni? Sotto quali punti di vista i loro percorsi di mobilità si differenziano da quelli dei centro-settentrionali e dei meridionali che non sono emigrati? In che modo queste differenze cambiano in base al genere e al titolo di studio?

Il lavoro è organizzato in sette paragrafi. A questo introduzione seguono due paragrafi teorici: il primo (par.2) descrive brevemente le caratteristiche del dualismo economico tra Nord e Sud e le sue implicazioni sulla mobilità sociale, mentre il par.3 si concentra sugli immigrati interni e su quello che sappiamo dei loro percorsi occupazionali nel Centro-nord. Il paragrafo 4 presenta i dati, le variabili e la strategia empirica utilizzata. I paragrafi 5 e 6 espongono i risultati empici e, infine, il paragrafo 7 propone alcune considerazioni conclusive.

\section{Dualismo economico e mobilità sociale}

Verso la fine degli anni Cinquanta, quando 1'Italia era un paese relativamente agricolo che si stava appena avviando verso il processo di industrializzazione, gli studi di stratificazione sociale avevano mostrato che nel nostro Paese il tasso di mobilità era inferiore rispetto a quello dei paesi più industrializzati. Lo studio di Livi (1950) condotto nel 1949 ha mostrato che il tasso di mobilità sociale in Italia era pari al 16\%. Utilizzando i dati di una survey condotta dalla DOXA tra il 1963 e il 1964, Lopreato (1965) ha mostrato che solo l' $8 \%$ degli italiani era riuscito a migliorare la propria posizione sociale, contro il 45\% degli svizzeri, il 39\% dei francesi, il 33\% degli statunitensi e il 29\% dei tedeschi.

Proprio in quei decenni, però, l'Italia ha cessato di essere un paese per molti aspetti sottosviluppato e prevalentemente agricolo, per diventare una nazione che si apprestava a entrare nel ristretto gruppo dei paesi industriali più avanzati. In questo periodo storico, che è comunemente definito miracolo economico (o boom economico), la struttura sociale ed economica del nostro paese è stata rivoluzionata: la popolazione agricola, che era la maggioranza alla fine della guerra, è diminuita drasticamente, mentre il numero di operai e di impiegati è cresciuto a ritmi accelerati. In che modo questi cambiamenti hanno influenzato i percorsi di mobilità sociale degli italiani?

Diverse ricerche hanno mostrato che l'ereditarietà sociale è diminuita con la generazione nata nell'immediato secondo dopoguerra, e nei decenni successivi le possibilità di mobilità sociale non hanno più raggiunto i livelli dello "scossone meritocratico" degli anni del miracolo economico (Barone 2012, p. 90). L'aumento delle opportunità di mobilità sociale degli anni Cinquanta e Sessanta non ha coinvolto in maniera indistinta tutte le classi sociali ma, analogamente a quanto è avvenuto per le disuguagliane di istruzione, ha riguardato soprattutto le classi agricole (Barone 2012). 
Lo sviluppo economico degli anni Cinquanta e Sessanta, così imponente da apparire miracoloso, ha avuto luogo spontaneamente, come un processo "dal basso", poco strutturato e per nulla pianificato. Per questo motivo il boom economico non ha solo significato sviluppo e miglioramento generalizzato delle condizioni di vita, ma ha anche aggravato diversi squilibri da tempo presenti nella struttura socio-economica italiana, tra i quali la "questione meridionale" e il dualismo economico e sociale tra Nord e Sud (Castronovo 2010; Barbagallo 2013). Spesso, infatti, si dimentica che il miracolo economico è stato un fenomeno soprattutto settentrionale: i principali settori dell'economia in espansione, i capitali, le capacità professionali e le aziende esportatrici della nazione erano concentrate - con rare eccezioni - nelle regioni nord-occidentali, e proprio queste regioni sono state coinvolte in misura maggiore dal processo di modernizzazione, dal consumismo di massa, dai cambiamenti della famiglia e dal mutamento degli stili di vita. Durante il boom economico la società meridionale ha invece continuato a essere prevalentemente rurale, povera e tradizionale. Le piccole industrie nate nelle città meridionali, infatti, hanno presto avuto la peggio di fronte alla concorrenza dei beni di consumo prodotti al Nord, e non sono state in grado di garantire posizioni lavorative stabili e tutelate ai loro dipendenti.

Anche grazie alla celebre ricerca svolta da Banfield (1958), il Mezzogiorno contadino e arretrato degli anni Cinquanta e Sessanta è stato oggetto di attenzione e interesse di un importante dibattito scientifico internazionale (Pizzorno 1967; Ferragina 2009); un dibattito che, nei decenni successivi, è andato via via scemando. Oltre a Banfield, molti altri studiosi stranieri si sono occupati delle comunità rurali del Sud dell'Italia e la "scoperta sociologica" del Mezzogiorno (Barbagallo 2013, p.151) ha mostrato la complessità dello sviluppo italiano degli anni del boom economico, dove modernità e arretratezza coesistevano all'interno di una stessa nazione.

A partire dal secondo dopoguerra la struttura occupazionale delle due aree si è quindi evoluta in maniera diversa: nel Centro-nord sono cresciute le occupazioni nel settore industriale e dei servizi, mentre nel Mezzogiorno questo sviluppo è stato più contenuto e la quota di braccianti agricoli - che sono praticamente scomparsi nel resto della penisola - è rimasta consistente (Cobalti e Schizzerotto 1994). La differenza tre le strutture occupazionali delle due aree è così evidente che, se si definisce industrializzata una società con una quota di addetti all'agricoltura non superiore al 10\% (Erikson e Goldthorpe 1992), allora si dovrebbe concludere che le regioni meridionali sono rimaste preindustriali fino al 1996, mentre quella del Centro-nord era industrializzata già nel 1967 (Panichella, 2014). Nel 1970, infatti, solo 1'8,1\% della popolazione centrosettentrionale era inclusa in una delle due classi agricole, a fronte del 23,8\% della popolazione meridionale ${ }^{2}$. È chiaro che queste differenze possono aver influenzato in maniera determinate $\mathrm{i}$ percorsi di mobilità sociale dei centro-settentrionali e dei meridionali.

\footnotetext{
${ }^{2}$ Nostra elaborazione sui dati ILFI (si veda par.4).
} 
Non bisogna poi dimenticare che le regioni centro-settentrionali non solo hanno conosciuto una crescita quantitativa del lavoro operaio e impiegatizio superiore a quello delle regioni del Sud, ma che il divario è stato anche qualitativo, dato che i posti di lavoro nell'industria e nei servizi del Centro-nord sono caratterizzati da un minore grado di precarietà e da mansioni più qualificate (Pugliese e Rebeggiani 2004). Un discorso analogo vale per il lavoro nel settore agricolo. Nelle aree rurali del Centro-nord, come i territori dell'arco alpino e alcune aree montane o collinari appenniniche della Toscana e delle Marche, l'esodo rurale si è accompagnato a uno sviluppo di attività extragricole e gli investimenti e i processi di modernizzazione dell'agricoltura sono stati relativamente elevati. Al contrario, le zone agricole del Sud - e in particolare le aree montane e collinari dell'entroterra - sono rimaste esclusivamente agricole, poco adatte alla localizzazione dell'agricoltura moderna, e presentano ancora oggi una struttura agraria basata sull'assoluto predominio delle piccole imprese contadine (Rossi-Doria 1982). Le difficoltà dell'agricoltura meridionale sono poi aggravate dalla cosiddetta povertà naturale del Mezzogiorno, ovvero dalla particolare conformazione fisica delle regioni del Sud e nella scarsa fertilità dei suoi terreni agricoli (Fortunato 1955). È ragionevole pensare che questa differenza qualitativa riguardi anche i lavori nel settore del commercio e dell'artigiano, vista l'ampia eterogeneità del lavoro autonomo in Italia (Barbieri 1999).

In che modo le differenze (quantitative e qualitative) delle due strutture occupazionali hanno influenzato i percorsi di mobilità sociale dei meridionali e dei centro-settentrionali? La questione non è stata affrontata nello specifico dalle ricerche più recenti sulla mobilità sociale, anche se la letteratura sul tema è concorde nel ritenere le che la regione di residenza (Centro-nord o Sud) rappresenta un importante fattore di strutturazione delle disuguaglianze sociali, accanto alla classe sociale di appartenenza, il genere e la generazione (Schizzerotto 2002, p. 362). Cobalti e Schizzerotto (1994), analizzando i dati Indagine nazionale sulla mobilità sociale del 1985, hanno mostrato che nel Centro-nord i passaggi dalla società agricola a quella industriale e da questa a quella post-industriale sono chiaramente visibili, mentre nel Mezzogiorno è presente un più alto tasso di immobilità intergenerazionale delle classi agricole e un ridotto accesso alla borghesia (imprenditori, dirigenti e liberi professionisti) da parte di soggetti provenienti da classi sociali inferiori. Questi risultati sono stati confermati anche da Barone (2012), che ha studiato le opportunità di mobilità sociale intergenerazione differenziando il campione dell'indagine Istat Multiscopo (2003) in base al genere e alla regione di residenza. I risultati ottenuti confermano che il divario territoriale per quel che riguarda le possibilità di ascesa sociale è notevole: gli uomini del Centro-nord fanno carriera più spesso, mentre nel Sud Italia le possibilità di mobilità sociale sono ridotte perché l'apertura di piccole imprese e la creazione di posti di lavoro qualificati nel settore terziario sono meno diffuse. 


\section{La mobilità sociale degli immigrati interni}

Quando si parla di differenze tra Mezzogiorno e Centro-nord si è inevitabilmente costretti a prendere in considerazione uno dei fenomeni più importanti della storia recente della popolazione del nostro paese: le migrazioni interne ${ }^{3}$. Il divario tra Nord e Sud è stato alla base di un rimescolamento senza precedenti della popolazione italiana: una volta concluso il periodo di riassestamento postbellico, e svanite le speranze di un cambiamento sociale basato sulla riforma agraria, gli spostamenti territoriali dal Sud verso il Centro-nord sono cresciuti a un ritmo superiore a quello di ogni altra fase della storia d'Italia. Gli anni del miracolo economico sono stati anche gli anni della grande migrazione interna; gli anni in cui molti meridionali hanno abbandonato il mondo, apparentemente immutabile, dell'Italia contadina per iniziare una nuova vita nelle città ricche e industrializzate del Centro-nord.

Negli anni Cinquanta e Sessanta le città settentrionali erano impreparate a gestire un tale afflusso, e i nuovi arrivati hanno dovuto affrontare grandi difficoltà nel trovare la casa e un lavoro, nell'inserire i figli a scuola e nell'allacciare rapporti di buon vicinato. Ben presto le condizioni di vita degli immigrati iniziano a essere osservate dalle inchieste della stampa e di molti altri osservatori. Come spesso accade, per prima cosa si osservano le situazioni più estreme e degradate: "Corea degli immigrati" o "gente che aveva perduto una guerra" (Alasia e Montaldi 1960) erano solo alcune delle definizioni comunemente usate per descrivere le condizioni di vita degli immigrati meridionali nelle regioni del Centro-nord. Si è così creato un importante dibattito pubblico e scientifico sul problema della difficile integrazione degli immigrati meridionali, un dibattito che ha contribuito in maniera determinante alla formazione $\mathrm{e}$ al consolidamento della sociologia italiana degli anni Cinquanta e Sessanta, rinata dopo essere stata cancellata dalla lunga stagione dell'egemonia dello storicismo.

Gli immigrati interni del boom economico erano prevalentemente giovani, poco istruiti, provenienti dalle aree rurali del Sud. Il loro inserimento nel mercato del lavoro non si è configurato come un'immediata e definitiva sistemazione nel comparto manifatturiero, in particolare nella grande industria, ma si è evoluto attraverso percorsi lavorativi per lo più di tipo orizzontale, molto dinamici, in cui la prima tappa è spesso costituita da un'occupazione nel settore edile (Fofi 1964; Reyneri 1979). L'arrivo dei lavoratori meridionali ha avuto un carattere prevalentemente sostitutivo-complementare e non concorrenziale rispetto alla forza lavoro locale, poiché la gran parte degli immigrati è andata a ricoprire i posti di lavoro rifiutati con sempre maggiore decisione dalla classe operaia centro-settentrionale (Ascoli 1979). Gli immigrati interni, quindi, non solo hanno dominato l'occupazione operaia nelle grandi città industriali del Nord ma, analogamente a quanto avviene negli ultimi decenni con gli immigrati provenienti da altri paesi (Ambrosini 2005; Reyneri e Fullin 2011; Panichella et al. 2021), si sono anche concentrati nelle posizioni più svantaggiate del lavoro manuale.

\footnotetext{
${ }^{3}$ Per una rassegna degli studi sulle migrazioni interne si veda Panichella (2014).
} 
I movimenti migratori interni dal Sud verso il Centro-nord hanno iniziato a diminuire a cavallo tra gli anni Settanta e Ottanta, fino a toccare il punto più basso nel 1994, quando "solo" 92.292 (comunque pari a 4 persone ogni 1.000 abitanti) si sono trasferite dal Sud verso il Centro-nord (Mocetti e Porello 2010; Panichella, 2014; Impicciatore e Strozza, 2016).

Gli spostamenti dal Sud hanno ripreso vigore nella seconda metà degli anni Novanta fino al 2000, ma senza tornare ai livelli della grande migrazione del boom economico (Livi Bacci 2007). Negli ultimi decenni sono cambiate anche le caratteristiche individuali degli immigrati interni, dato che sempre più spesso si spostano coloro che hanno un titolo di studio elevato (Panichella, 2009; 2013) Tuttavia, più che confermare un reale cambiamento della composizione interna del flusso migratorio, recenti analisi hanno mostrato che i laureati e i diplomati sono sempre emigrati, quello che è realmente cambiato è il fatto che i meno istruiti hanno ridotto la propensione a spostarsi (Panichella 2012). Lo stesso di può dire per le aree di partenza e di destinazione dei flussi migratori: negli anni del boom economico i meridionali partivano soprattutto dalle aree rurali del Mezzogiorno e si dirigevano verso le aree industriali del Nord-ovest, mentre negli ultimi anni le migrazioni interne si caratterizzano ma da una pluralità di aree di partenza e di destinazione.

Le migrazioni interne dal Sud verso il Centro-nord sono state un fenomeno molto importante nella recente storia italiana, per cui è importante studiare gli effetti che $\mathrm{i}$ movimenti di popolazione hanno avuto nel medio e lungo periodo sui percorsi di mobilità sociale degli immigrati e delle loro famiglie (Ginsborg 1989; Panichella, 2014). La questione è interessante perché, come si è visto nel paragrafo precedente, gli immigrati interni hanno abbandonato una struttura di classe caratterizzata da un'eccedenza di manodopera nel settore agricolo, per inserirsi in un contesto che offriva più posizioni nella classe operaia urbana e in quella impiegatizia. In altre parole, emigrando verso il Centro-nord, i meridionali hanno abbandonato un contesto arretrato e rurale per inserirsi in una struttura occupazionale più sviluppata e industrializzata, che garantiva migliori opportunità occupazionali sia nel settore industriale che in quello terziario. Inoltre, si tratta di una migrazione in larga parte definitiva. Molti meridionali che hanno abbandonato il Mezzogiorno non sono tornati nel loro luogo di origine: si sono insediati stabilmente nel Centro-nord, hanno formato una famiglia e hanno avuto figli (Guetto e Panichella, 2013), quella che gli studiosi chiamano una "seconda generazione" di immigrati. Le ricerche empiriche sul tema hanno confermato che le migrazioni interne si sono associate a una consistente mobilità sociale, che ha riguardato soprattutto lo spostamento dalle classi agricole a quelle operaie, ma al tempo stesso i meridionali che si sono spostati al Nord sono stati penalizzati per quel nell'accesso al lavoro autonomo (Panichella, 2014), che rappresenta il canale di mobilità sociale ascendente più diffuso nella società italiana, anche tra gli immigrati (Panichella et al., 2021). 


\section{Dati, variabili e metodo}

\section{Dati}

L'obiettivo di questo lavoro è studiare i percorsi di mobilità sociale degli immigrati interni, mettendoli a confronto con quello dei centro-settentrionali e dei meridionali che non sono emigrati. A tal fine sono stati analizzati i dati dell'Indagine Longitudinale sulle Famiglie Italiane (da ora in avanti ILFI, cfr. A. Schizzerotto (2002). I dati della ricerca ILFI, aggiornati al 2005, contengono informazioni dettagliate sui percorsi geografici, scolastici, professionali e familiari di un campione rappresentativo della popolazione italiana. Le analisi si concentrano sugli intervistati nati tra il 1930 e il 1975, considerando come finestra osservativa 15-60 anni. Dopo aver eliminato i casi mancanti, il campione analitico include 5,818 individui.

\section{Variabili}

\section{La mobilità geografica}

La variabile relativa alla provenienza geografica include quattro diverse modalità: a) centro-settentrionali, b) meridionali statici, c) emigrati e d) tornati. La prima categoria include gli intervistati che sono nati nel Centro-nord e che risiedevano in queste regioni al compimento del quindicesimo anno di età. Mentre i centro-settentrionali rimangono in questa categoria per tutto il tempo di osservazione, i meridionali possono cambiare modalità nel corso del tempo. Al momento della prima osservazione - che, ricordiamo, corrisponde al compimento del quindicesimo anno di età - tutti i meridionali sono considerati statici. Quando uno statico si trasferisce al Centro-nord diventa un meridionale emigrato. Questi ultimi passano alla categoria dei tornati se fanno ritorno nel Mezzogiorno ${ }^{4}$.

\section{La classe sociale}

Per descrivere i percorsi di mobilità sociale, è stata utilizzata l'aggregazione di classe che sviluppata negli anni settanta da Goldthorpe e dai suoi collaboratori, che nel decennio successivo è stata rivisitata e adattata al caso italiano (Cobalti e Schizzerotto 1994). I soggetti inclusi nel campione Ilfi sono stati suddivisi in sei diverse classi sociali: 1) la borghesia (BOR); 2) la classe media impiegatizia (CMI); 3) la piccola borghesia urbana (PBU); 4) la piccola borghesia agricola (PBA); 5) la classe operaia urbana (COU); 6) la classe operaia agricola (COA). Queste sei classi possono essere ordinate gerarchicamente in tre gruppi: la borghesia è la classe superiore; la classe impiegatizia e le due piccole borghesie formano la classe media; infine, la classe operaia agricola e la classe operaia urbana formano le classi basse. Questo lavoro considera mobilità ascendente i passaggi dalle tre classi medie (cmi, pbu e pba) alla borghesia e

\footnotetext{
${ }^{4}$ A causa della scarsa numerosità campionaria, i risultati riguardanti gli immigrati che hanno compiuto altri movimenti dopo il ritorno non sono mostrati.
} 
quelli dalle classi basse (cou e coa) verso le classi medie o verso la borghesia. Al contrario, per mobilità discendente si intende il passaggio a una posizione più bassa nel sistema di stratificazione sociale, come quello dalla borghesia verso le altre classi oppure dalle classi medie verso quelle operaie. Infine, per mobilità orizzontale si intende il passaggio da una posizione sociale a un'altra dello stesso rango, quindi riguarda la mobilità all'interno delle classi medie e all'interno delle classi basse.

\section{La desiderabilità sociale}

Un'altra misura che sarà presa in considerazione in questo lavoro è il grado di desiderabilità sociale (o di "prestigio sociale") dell'occupazione svolta, riferito alle sue diverse dimensioni: la posizione dell'occupazione nell'ordinamento gerarchico dell'organizzazione di cui è parte; il grado di autonomia del lavoratore nello svolgimento dei suoi compiti; le opportunità di reddito e di carriera associate all'occupazione. Tra le diverse scale di questo tipo, quella più frequentemente utilizzata è stata costruita da De Lillo e Schizzerotto (1985). Per costruirla, a un campione di individui rappresentativo della popolazione italiana, è stato chiesto di ordinare una serie di occupazioni in base alla loro maggiore o minore desiderabilità: questi ordinamenti individuali sono poi stati trasformati in un ordinamento generale, che attribuisce un punteggio a ogni occupazione presa in considerazione. Il punteggio minimo della scala è 9,97 , che corrisponde alle occupazioni manuali a bassa qualificazione in posizioni dipendenti (per esempio lavoratori manuali del settore primario), mentre quello massimo è 90,2, che corrisponde alle occupazioni relative all'alta dirigenza dello stato (per esempio ambasciatore, prefetto, ecc.).

\section{Metodo}

La strategia empirica è divisa in due parti. La prima descrive mobilità sociale degli immigrati interni, dei centro-settentrionali e dei meridionali attraverso l'analisi delle tavole di mobilità. Si tratta di tabelle a doppia entrata che suddividono gli individui in base alla loro classe sociale di partenza e di destinazione. Ogni cella della tabella, quindi, rappresenta una specifica combinazione di origine e destinazione. La seconda parte ha due obiettivi: a) controllare la robustezza dei risultati e i possibili effetti di composizione; b) studiare come gli effetti della migrazione interna sui destini sociali cambiano in base al genere e al titolo di studio. Dato che questi obiettivi richiedono un'elevata numerosità campionaria, in questa seconda parte al posto delle tavole di mobilità sono stati stimati una serie di Linear Regression Panel Models con effetti random. Infine, sempre per ridurre l'incertezza delle stime, questi modelli non sono stati utilizzati per studiare la transizione in specifiche traiettorie di mobilità sociale. Al contrario, essi sono stati utilizzati per studiare il rapporto tra il punteggio di prestigio occupazionale degli individui rispetto a quello della famiglia di origine. L'interpretazione del rapporto è semplice. Esso va da 0 a infinito: il valore 1 indica che 
il soggetto ha raggiunto un prestigio sociale identico a quello dei genitori; valori superiori a 1 indicano un miglioramento rispetto alla famiglia di origine; infine, i valori compresi tra 0 e 1 indicano che il soggetto ha un punteggio di desiderabilità sociale inferiore rispetto a quello di partenza.

\section{La mobilità sociale intergenerazionale degli immigrati interni}

La prima parte dell'analisi empirica studia la mobilità intergenerazionale, che si riferisce ai cambiamenti di posizione sociale nel passaggio dalla generazione dei genitori a quella dei figli ${ }^{5}$. Il paragrafo 5.1 studia si concentra solo sugli immigrati interni e studia la loro mobilità sociale prima e dopo lo spostamento territoriale, mentre il paragrafo successivo (par 5.2) mette a confronto gli immigrati con i centrosettentrionali e con i meridionali statici.

\section{Le origini sociali e i destini occupazionali prima e dopo la migrazione}

Nella tabella 1 è riportata la mobilità dalla classe di origine alla classe dell'occupazione svolta due anni prima dello spostamento territoriale, e contiene i valori assoluti e le percentuali di riga. La migrazione interna è spesso associata con il primo inserimento nel mercato del lavoro, per cui a differenza delle classiche tavole di mobilità sociale in questa tavola la classe sociale di destinazione comprende anche i non occupati (disoccupati e inattivi).

\section{[circa qui tabella 1]}

In effetti, due anni prima della migrazione ben il 59,4\% degli immigrati aveva un'occupazione. Non tutti i meridionali, però, si sono spostati senza aver mai svolto alcuna attività lavorativa nel Mezzogiorno: la percentuale di individui mai occupati prima della migrazione è più bassa per gli originari delle due classi agricole, mentre è più alta per gli originari della classe media impiegatizia e della borghesia. Infatti, il $18,8 \%$ degli originari della piccola borghesia agricola e, addirittura, il 38,7\% degli originari della classe operaia agricola sono entrati nella classe operaia urbana già prima della migrazione nel Centro-nord.

Questo risultato mostra ancora una volta come per le classi agricole la mobilità intersettoriale all'interno del Mezzogiorno abbia spesso preceduto quella territoriale verso il Centro-nord (Reyneri 1979). Negli anni del miracolo economico si è avviata una migrazione interna al Mezzogiorno stesso, perché la crisi dell'agricoltura ha spinto

\footnotetext{
${ }^{5}$ Dati gli obiettivi descrittivi del paragrafo, si è scelto di riportare solo le misure di mobilità assoluta. Dunque, bisogna considerare che nelle regioni centro-settentrionali l'industrializzazione ha provocato una forte contrazione delle due classi agricole e una crescita della classe operaia urbana, per cui in queste regioni la mobilità dei figli delle classi agricole è stata (almeno in parte) inevitabile (Pisati 2002). A tal proposito sono stati calcolati gli odds ratio generalizzati e si è costruita una tavola di mobilità relativa per i centro-settentrionali, gli statici e gli immigrati interni. Anche i valori degli odds ratio generalizzati, che non sono stati riportati per ragione di spazio ma che sono disponibili su richiesta, confermano la robustezza dei risultati presentati nei prossimi due paragrafi.
} 
molti migranti prima dalle zone rurali più depresse verso le grandi città meridionali, e poi dal Sud verso il Centro-nord (Mottura e Pugliese 1975). Per questo motivo i membri delle classi agricole si sono inizialmente inseriti nella classe operaia urbana del Meridione e, pur di non far ritorno alle aree rurali, hanno deciso di emigrare nel Centronord quando queste nuove possibilità lavorative sono venute meno. Reyneri (1979, p.188) ha scritto in proposito: "ai campi non si ritorna tanto facilmente una volta fatta l'abitudine a migliori condizioni salariali e lavorative".

La mobilità sociale precedente alla migrazione interna ha riguardato, in misura minore, anche l'accesso alla classe media impiegatizia: il 14,7\% degli originari della borghesia, il 12,3\% della piccola borghesia urbana e 1'8.9\% della piccola borghesia agricola ha svolto un lavoro impiegatizio prima di emigrare al Centro-nord. Anche il tasso di immobilità, ovvero la percentuale di individui che si trova nella stessa classe di origine, è particolarmente alta tra gli originari della classe media impiegatizia $(16,7 \%)$.

La migrazione interna ha accentuato e rinforzato queste dinamiche: dopo il trasferimento territoriale le classi agricole diminuiscono ulteriormente, a favore della classe operaia urbana e della classe impiegatizia. La tabella 2 è una tavola di mobilità che mette a confronto la classe sociale di origine con quella dell'occupazione svolta due anni dopo la migrazione: si vede come dopo la migrazione la classe operaia agricola e la piccola borghesia agricola abbiano continuato a "svuotarsi", mentre è particolarmente elevata la quota di individui che si sono inseriti nella classe operaia urbana. Gli unici immigrati che, invece, hanno avuto buone possibilità di conservare la propria classe sociale di origine nelle regioni di arrivo sono stati proprio i membri della classe operaia urbana e quelli della classe media impiegatizia.

\section{[circa qui tabella 2]}

Un altro risultato interessante riguarda il tasso di non occupazione. La percentuale di intervistati senza occupazione a due anni dallo spostamento territoriale è alta tra gli originari della borghesia mentre è piuttosto ridotta per chi proviene dalle classi medie e basse. Questa differenziazione sembra essere la conseguenza dei diversi modi con cui i meridionali tentano di entrare nel mercato del lavoro centro-settentrionale: mentre coloro che provengono dalle classi alte emigrano e ricercano lavoro direttamente nella zona di arrivo, chi proviene dalle classi medie e basse cerca di minimizzare i rischi della migrazione decidendo di spostarsi solo quando sono disponibili possibilità occupazionali certe (Panichella 2012; 2014).

Se si analizza la mobilità sociale degli immigrati per un periodo più lungo, si trovano sostanzialmente gli stessi risultati (risultati disponibili su richiesta). Anche questi andamenti confermano che, una volta giunti nel Centro-nord, una buona parte dei meridionali immigrati è entrata nella classe operaia urbana e nella classe media impiegatizia. Esiste, peraltro, una chiara dicotomia: da un lato, gli originari delle classi basse - in particolare quelle agricole - si sono inseriti nella classe operaia centrosettentrionale; dall'altro gli originari della borghesia e della classe media impiegatizia 
hanno avuto accesso a lavori impiegatizi (Panichella, 2014). Da questo punto di vista è importante sottolineare che per molti meridionali emigrati l'impiego pubblico ha influenzato in modo decisivo le loro possibilità di ascesa sociale: da un lato, coloro che sono entrati nella pubblica amministrazione hanno migliorato la propria posizione con la stessa velocità dei lavoratori centro-settentrionali (pubblici e privati); dall'altro, i meridionali che sono emigrati ma che non hanno avuto accesso a un posto di lavoro pubblico impiego sono i più lenti. I risultati (disponibili su richiesta) mostrano gli immigrati che sono entrati nel pubblico impiego, quindi, hanno avuto le stesse possibilità di carriera degli autoctoni. centro-settentrionali e il 33\% circa dei meridionali che non si sono spostati. Anche Banfield (1976), analizzando i rapporti di classe tra gli abitanti di Montegrano, osserva che per i braccianti il pubblico impiego rappresenta un'opportunità importante di ascesa sociale, mentre sono scarse le possibilità di imparare un mestiere e diventare artigiani. "Con il diploma di scuola media -scrive Banfield- un ragazzo può diventare carabiniere (basta formalmente anche la quinta elementare ma data l'affluenza ai concorsi di ammissione, il diploma di scuola media è praticamente necessario). Riuscire in questo è un passo notevole per un contadino; come carabiniere sarebbe ben vestito e ben nutrito, e la paga è di 500 lire a giorno abbastanza da permettergli di mantenere la madre o costituire una dote per la sorella" (Banfield 1976, p.93).

\section{Gli immigrati a confronto}

Questo paragrafo confronta la mobilità sociale degli immigrati con quella dei centrosettentrionali e dei meridionali statici al momento dell'ultima rilevazione dell'indagine Ilfi $(2005)^{6}$.

\section{[circa qui tabella 3]}

La prima differenza rilevante il Centro-nord e il Sud riguarda le classi agricole: nel Centro-nord una larga parte degli originari della classe operaia agricola e della piccola borghesia agricola è entrata nella classe operaia urbana (rispettivamente 61,2\% e $46,1 \%)$, mentre solo una piccola parte è rimasta immobile $(11,1 \%$ e 17,1$)$. Nel Mezzogiorno, al contrario, le percentuali di individui provenienti dalla classe operaia agricola e dalla piccola borghesia agricola che si sono inseriti nella classe operaia sono inferiori $(43,9 \%$ e $32,1 \%)$, e i tassi di immobilità di queste due classi sono più alti (rispettivamente $33,7 \%$ e 22,8 ).

Tra gli immigrati la percentuale di individui mobili è superiore rispetto a quella dei centro-settentrionali e degli statici, per via di due fattori. In primo luogo, perché è particolarmente bassa la quota di immigrati immobili $(10,0 \%)$ nella piccola borghesia urbana. Dopo il trasferimento territoriale, chi proviene dalla piccola borghesia urbana ha

\footnotetext{
${ }^{6}$ Le analisi sono state replicate considerando la condizione occupazionale a 35, 40 e 45 anni (si veda nota 6). I risultati ottenuti sono sostantivamente identici a quelli presentati.
} 
più difficoltà a rimanere nella classe sociale di origine. Infatti, sembra difficile che $\mathrm{i}$ figli dei piccoli artigiani e commercianti decidano di emigrare per fare lo stesso mestiere nelle regioni del Centro-nord. Se i figli della piccola borghesia urbana decidono di fare un mestiere simile a quello dei genitori, è molto più ragionevole rimanere nel luogo di origine e continuare a lavorare nell'attività familiare, sfruttando le risorse economiche e relazionali già consolidate, piuttosto che affrontare i costi dello spostamento territoriale e quelli legati all'avviamento di una nuova attività nel Centronord. Il secondo fattore sono i bassi tassi di immobilità delle due classi agricole. La migrazione interna, infatti, ha comportato anche una mobilità intersettoriale dal settore primario al settore secondario, e molti meridionali provenienti dalle classi agricole e dopo lo spostamento territoriale sono entrati nelle grandi fabbriche del Nord.

\section{Emigrazione e mobilità sociale per genere e per titolo di studio}

Dopo aver descritto la mobilità sociale intergenerazionale attraverso la descrizione delle tavole di mobilità sociale, in questo paragrafo metteremo a confronto gli immigrati interni, dei meridionali e dei centro-settentrionali considerando anche le differenze di genere, di coorte e di istruzione. Chi sono gli immigrati interni più penalizzati? La limitata numerosità campionaria non permette di analizzare questi elementi di eterogeneità con le tavole di mobilità. Come abbiamo anticipato, la scarsa numerosità campionaria non permette di costruire delle tavole di mobilità differenziando la popolazione degli immigrati meridionali al suo interno. Per questo motivo, in questo paragrafo sono presentati una serie di modelli lineari panel con effetti random che hanno l'obiettivo di vedere se le possibilità di mobilità delle donne sono diverse da quelle degli uomini, oppure se cambiano in base al titolo di studio raggiunto. In questi modelli la variabile dipendente è il rapporto tra il punteggio di prestigio occupazionale della famiglia di origine e quello dell'intervistato.

[circa qui tabella 4]

Per quel che riguarda il genere, i risultati confermano un risultato ben noto in letteratura (Ballarino e Panichella, 2021), ovvero che la mobilità geografica ha un effetto positivo per gli uomini e negativo per le donne, e questo è confermato anche quando si considera la mobilità di ritorno. I coefficienti relativi alla mobilità Sud-Nord stimati sono infatti 0,10 per gli uomini e $-0,02$ per le donne. Inoltre, è interessante notare come gli uomini emigrati hanno un vantaggio non solo rispetto ai centro settentrionali ma anche rispetto ai meridionali statici, mentre le donne che si sono spostate sono svantaggiate rispetto a entrambi i gruppi. Questo risultato è probabilmente influenzato da diversi fattori, legati sia al processo selettivo delle donne immigrate, sia alle interrelazioni tra eventi famigliari e spostamento territoriale (Impicciatore e Panichella, 2019). Infatti, mentre le donne che non sono emigrate spesso ricadono nell'inattività dopo la nascita di un figlio, le immigrate, spinte da un maggior bisogno economico, insistono nella ricerca di un 
lavoro, anche di bassa qualità, purché compatibile con la cura dei figli (Panichella, 2014). Di conseguenza, il gruppo delle emigrate comprende una quota di persone che si è inserita nel mercato del lavoro - oppure, specularmente, che non è uscita dalla forza lavoro - per ammortizzare i costi della migrazione e contribuire al reddito familiare.

\section{[circa qui tabella 5]}

Differenziando il campione in base al genere e al titolo di studio, emerge che la migrazione interna influenza positivamente la mobilità sociale ascendente per gli uomini, specialmente quando hanno titoli di studio bassi. Al contrario, per le donne la migrazione interna è vantaggiosa solo per coloro che hanno un'istruzione medio-alta, mentre per coloro che hanno un titolo di studio basso sono sistematicamente svantaggiate. Dunque, se è vero che gli immigrati meridionali uomini poco istruiti si sono inseriti nei piani bassi della struttura occupazionale, è altrettanto vero che con la migrazione interna questi hanno migliorato la posizione sociale dei loro genitori, mentre l'effetto positivo per gli uomini laureati o diplomati è inferiore. Sono invece le donne poco istruite quelle ad aver avuto la penalizzazione più alta dal punto di vista delle possibilità di mobilità sociale.

\section{Conclusione}

\section{La mobilità sociale degli immigrati interni}

È ora possibile rispondere alle domande di ricerca formulate nel paragrafo introduttivo di questo lavoro. Iniziamo dalla prima: quali sono stati i percorsi di mobilità intergenerazionale degli immigrati interni? La migrazione interna dal Sud verso il Centro-nord ha comportato una notevole mobilità intergenerazionale, favorendo l'ingresso nella classe operaia urbana e nella classe media impiegatizia. In generale, si possono individuare tre tipi di percorso degli immigrati: a) alla cou, che ha riguardato $i$ meridionali originari delle classi inferiori, in particolar modo i figli dei contadini e dei braccianti; b) alla cmi, che invece ha riguardato soprattutto gli originari della borghesia e della classe media impiegatizia; c) misti, che hanno coinvolto degli originari della piccola borghesia e della classe operaia urbana. In questi casi, infatti, molti individui sono entrati nella classe operaia, ma è consistente anche la quota di immigrati che si sono inseriti nella classe media impiegatizia.

Se si applica la definizione standard di mobilità ascendente, considerando come tale solo il passaggio dalle classi basse a quelle medio-alte e da quelle medie verso quelle alte, allora si può concludere che la migrazione interna non si è associata a migliori possibilità di mobilità intergenerazionale. Questo è particolarmente vero per gli originari delle due classi agricole, perché il loro inserimento nella struttura occupazionale centro-settentrionale è avvenuto ai livelli bassi, soprattutto all'interno della classe operaia urbana: si tratta quindi di mobilità sociale discendente per gli 
originari della piccola borghesia agricola e di mobilità orizzontale per gli originari della classe operaia agricola.

D'altra parte, classificare il passaggio dalle classi agricole alla classe operaia urbana come mobilità sociale discendente potrebbe essere problematico, poiché l'abbandono del lavoro nelle campagne apporta di solito importanti miglioramenti delle condizioni di vita e di lavoro (Ginsborg 1989). Molti immigrati interni sono passati dal lavoro agricolo a quello industriale, e l'abbandono delle campagne ha rappresentato un importante miglioramento dello stile di vita, delle condizioni di lavoro, della stabilità dei redditi e del comfort abitativo (Lopreato 1967; Reyneri 1979; Panichella 2014). Inoltre, con la migrazione interna molti meridionali hanno anche migliorato la qualità della loro occupazione, perché il lavoro industriale nel Centro-nord è caratterizzato da un minor grado di instabilità e precarietà. Secondo altri, però, questi miglioramenti non possono essere considerati come mobilità ascendente: anche se il figlio di un contadino arrivato in città percepisce un reddito superiore a quello del padre, la sua autonomia e il suo controllo sulla propria vita lavorativa possono essere minori di quelli di cui godeva il padre (Heath 1981).

Veniamo alla seconda domanda: sotto quali punti di vista la mobilità sociale degli immigrati interni è diversa da quello dei centro-settentrionali e dei meridionali statici? La differenza principale rispetto ai meridionali non emigrati riguarda i minori tassi di immobilità delle due classi agricole: con la migrazione interna, gli originari della classe operaia agricola e della piccola borghesia si sono inseriti prevalentemente nella classe operaia centrosettentrionale, mentre tra gli statici originari di queste due classi è particolarmente alta la quota di immobili. Questo risultato mostra che la crisi del settore agricolo del Sud ha messo i meridionali (emigrati e statici) davanti a un'alternativa: da un lato, non emigrare e aumentare le possibilità di rimanere nella classe sociale di origine, ma con poche possibilità di salire verso l'alto e, per i figli della piccola borghesia agricola, con un rischio maggiore di scendere nella classe operaia agricola; dall'altro lato, emigrare al Centro-nord, al costo di annullare la possibilità di conservare la propria origine sociale, ma con più possibilità di inserirsi nella classe operaia urbana e nella piccola borghesia urbana. La struttura occupazionale dei meridionali è più rigida non solo ai "piani bassi", ma anche per quel che riguarda l'accesso alla classe media impiegatizia e alla borghesia. Infatti, gli immigrati interni provenienti da queste due classi hanno diminuito le possibilità di conservare la classe sociale di origine rispetto agli statici. Questo risultato è probabilmente legato al fatto che nel mercato del lavoro centro-settentrionale è più diffusa una mobilità discendente delle classi alte, per cui parte dei figli della borghesia inizia la carriera nelle classi medie, per poi risalire alla classe di origine. Invece nel Meridione i figli delle classi alte entrano più spesso direttamente nella loro stessa classe di origine. 


\section{Differenze di genere e di istruzione}

La terza domanda di ricerca è la seguente: in confronto ai lavoratori centro-settentrionali e agli statici, gli immigrati dal Sud sono rimasti svantaggiati, o hanno avuto le stesse possibilità di mobilità sociale? I risultati di questo studio hanno mostrato che la migrazione ha comportato dei vantaggi per gli uomini e degli svantaggi per le donne: infatti, mentre i primi in genere hanno migliorato la loro condizione lavorativa rispetto a quella dei loro genitori, le seconde sono sistematicamente svantaggiate rispetto alle centro-settentrionali e alle meridionali non emigrate.

La penalizzazione delle donne immigrate dipende dall'effetto congiunto di due fattori: la migrazione familiare e la necessità di ammortizzare i costi della migrazione. Anche per le migrazioni interne, la strategia migratoria più diffusa è quella al maschile, in cui è l'uomo a emigrare per prima, mentre la donna si sposta in un secondo momento, quando i costi della migrazione sono stati ammortizzati. Anche lo spostamento territoriale interno dal Sud verso il Centro-nord è avvenuto più come risposta alle esigenze lavorative degli uomini che a quelle delle donne (Panichella, 2014). Anche il trasferimento da un sistema economico arretrato, come quello del Mezzogiorno, verso uno più sviluppato e industrializzato ha comportato l'ingresso nel mercato del lavoro di donne che in precedenza non erano mai state occupate perché troppo giovani o per la mancanza di opportunità, la migrazione femminile è associata a sistematici svantaggi occupazionali in termini di qualità del lavoro e di possibilità di mobilità sociale. Dunque, dopo lo spostamento al Nord, molte donne emigrate sono state incoraggiate a trovare un'occupazione, anche di bassa qualità, per aumentare il reddito familiare.

Le possibilità di mobilità sociale degli immigrati interni non cambiano solo tra uomini e donne, ma anche tra istruiti e meno istruiti. Mentre gli uomini immigrati sono sempre avvantaggiati, indipendentemente dalla loro istruzione, tra le donne è presente uno svantaggio solo tra coloro che hanno un titolo di studio basso. È quindi possibile che il progetto migratorio al maschile descritto in precedenza abbia influenzato $i$ processi di integrazione delle donne poco istruite, poiché quelle più istruite sono emigrate in maniera più autonoma e indipendente rispetto ai vincoli familiari. Non bisogna poi dimenticare che molti meridionali sono arrivati nel Nord per frequentare l'università, e questo ha sicuramente facilitato il loro inserimento occupazionale: durante la frequenza dei corsi universitari, le donne e gli uomini meridionali hanno avuto la possibilità di stringere legami con i centro-settentrionali di buona estrazione sociale, creando i presupposti per un buon inserimento nel mercato del lavoro. La migrazione delle donne e degli uomini meno istruiti è molto diversa: questi hanno avuto una maggiore necessità di inserirsi velocemente nel mercato del lavoro centrosettentrionale, anche affidandosi ai legami della rete migratoria che, com'è ben noto in letteratura, sono più diffusi nei livelli bassi della struttura occupazionale. Questo tipo di migrazione ha spinto le donne meridionali poco istruite nelle posizioni lavorative più dequalificate, creando quindi gli svantaggi che sono persistiti per tutta la carriera 
lavorativa, e che con tutta probabilità hanno influenzato negativamente anche i percorsi educativi e occupazionali dei loro figli. 


\section{Bibliografia}

Ambrosini, M. (2005). Sociologia delle migrazioni. Il mulino.

Alasia, F., e Montaldi, D., (1960), Milano, Corea. Inchiesta sugli immigrati, Milano: Feltrinelli

Arum, R., e. Müller, W., (2004). Self-employment dynamics in advanced economies. The Reemergence of Self-Employment: A comparative study of self-employment dynamics e social inequality, 1-35.

Ascoli, U. (1979). Movimenti migratori in Italia (Vol. 96). Il mulino.

Ballarino, G., Panichella, N. (2021). Social origins, geographical mobility and occupational attainment in contemporary Italy. Genus, 77(1), 1-24.

Banfiled, E., (1958). The moral basis of a backward society. Glencoe, 111, 85.

Barbagallo, F., (2013), La questione italiana. Il Nord e il Sud dal 1860 a oggi, Bari, Laterza

Barbieri, P. (1999). Liberi di rischiare. Vecchi e nuovi lavoratori autonomi. Stato e mercato, 19(2), 281-308.

Barbieri, P., e Bison, I. (2004). Self-employment in Italy: scaling the class barriers. The Reemergence of Self-employment. A Comparative Study of Self-employment Dynamics e Social Inequality, 121-73.

Barone, C. (2012). Le trappole della meritocrazia, il Mulino.

Bison, I., (2002), Le opportunità di carriera, in Schizzerotto (2002).

Blau, P. M., e Duncan, O. D. (1967). The American occupational structure.

Castronovo, V. (2010). L'Italia del miracolo economico. Editori Laterza.

Cobalti, A. (1995). Lo studio della mobilità: metodi e prospettive dell'indagine sociologica. La Nuova Italia Scientifica.

Cobalti e Schizzerotto (1994), La mobilità sociale in Italia. Bologna, Il Mulino

Erikson, R., e Goldthorpe, J. H. (1992). The constant flux: A study of class mobility in industrial societies. Oxford University Press, USA.

Ferragina, E. (2009). Le teorie che non muoiono mai sono quelle che confermano le nostre ipotesi di base: cinquant'anni di familismo amorale. Meridiana, 265-287.

Fofi, F., (1964), L'immigrazione meridionale a Torino. Milano, Feltrinelli.

Fortunato, G., (1955), Povertà naturale del Mezzogiorno, in Caizzi, B., (1955), Antologia della questione meridionali, Milano, Edizioni Comunità.

Ginsborg, P. (1989). Storia d'Italia dal dopoguerra a oggi: Dal" miracolo economico" agli anni'80 (Vol. 2). Einaudi.

Granovetter, M. (1983). The strength of weak ties: A network theory revisited. Sociological theory, 1(1), 201-233.

Guetto, R., Panichella, N. (2013). Geographical mobility and reproductive choices of Italian men. European Sociological Review, 29(2), 302-315.

Heath, A. F. (1981). Social mobility (Vol. 5601). London: Fontana paperbacks.

Impicciatore, R., Panichella, N. (2019). Internal migration trajectories, occupational achievement and social mobility in contemporary Italy. A life course perspective. Population, Space and Place, 25(6), e2240. 
Impicciatore, R., Strozza, S. (2016). Internal and international migration in Italy. An integrating approach based on administrative data. Polis, 30(2), 211-238.

Lipset, S. M., e Bendix, R., (1959) Social Mobility in Industrial Societies, University of California Press Berkeley, CA.

Livi, L., (1950), Sur le mesure de la mobilite so-ciale," Population, V (January-March, 1950), 65- 76.

Livi Bacci, M., (2007), Ma c'è davvero una ripresa delle migrazioni sud-nord? in Neodemos (www.neodemos.it) Pubblicato il 19/12/2007

Lopreato, J. (1965). Social mobility in Italy. The American Journal of Sociology, 71(3), 311-314.

Lopreato, J. (1967). Peasants no more: Social class e social change in an underdeveloped society. Chandler Publishing Company.;

Merton, R.K., (1959). Social theory e social structure. Glenco, Illinois: The Free Press.

Mocetti, S., e Porello, C. (2010). Labour mobility in Italy: new evidence on migration trends. Bank of Italy Occasional Paper, (61).

Paci, M. (1982). La struttura sociale italiana. Il Mulino, Bologna.

Panichella, N. (2009). La mobilità territoriale dei laureati meridionali: vincoli, strategie e opportunità. Polis, 23(2), 221-246.

Panichella, N. (2012). Le migrazioni interne nel secolo scorso: vecchie e nuove forme a confronto. Stato e mercato, 32(2), 255-282.

Panichella, N. (2013). Migration strategies and occupational outcomes of southern Italian graduates. Journal of Modern Italian Studies, 18(1), 72-89.

Panichella, N. (2014). Meridionali al Nord: migrazioni interne e società italiana dal dopoguerra ad oggi. Il mulino.

Panichella, N., Avola, M., Piccitto, G. (2021). Migration, Class Attainment and Social Mobility: An Analysis of Migrants' Socio-Economic Integration in Italy. European Sociological Review.

Pizzorno, A. (1967), Familismo amorale e marginalità storica, ovvero perché non c'è niente da fare a Montegrano, in Quaderni di sociologia, n. 3, pp. 247-261

Pisati, M. (2000). La mobilità sociale. Il mulino.

Pugliese, E., e Rebeggiani, E. (2004). Occupazione e disoccupazione in Italia: dal dopoguerra ai giorni nostri (Vol. 129). Lavoro.

Reyneri, E. (1979). La catena migratoria. Il Mulino.

Reyneri, E., e Fullin, G. (2011). Labour market penalties of new immigrants in new e old receiving West European countries. International Migration, 49(1), 31-57.

Rossi-Doria, M. (1982). Scritti sul Mezzogiorno (Vol. 642). G. Einaudi.

Schizzerotto, A. (Ed.). (2002). Vite ineguali: disuguaglianze e corsi di vita nell'Italia contemporanea (Vol. 497). Il mulino.

Sorokin, P. A. (1927). Social mobility (Vol. 3). Taylor e Francis. 


\section{Tabelle}

Tabella 1 - tavola di mobilità intergenerazionale all'occupazione svolta due anni prima dello spostamento territoriale: frequenze assolute e percentuali di riga.

\begin{tabular}{|c|c|c|c|c|c|c|c|c|}
\hline \multirow{2}{*}{$\begin{array}{l}\text { Classe } \\
\text { sociale di } \\
\text { origine: }\end{array}$} & \multicolumn{7}{|c|}{ Classe sociale due anni prima della migrazione } & \multirow[b]{2}{*}{ Totale } \\
\hline & Non occ. & Borg & $\mathrm{Cmi}$ & Pub & $\mathrm{Pba}$ & Cou & Coa & \\
\hline & 23 & 1 & 5 & 0 & 0 & 4 & 1 & 34 \\
\hline \multirow[t]{2}{*}{ Bor } & 67.6 & 2.9 & 14.7 & 0.0 & 0.0 & 11.8 & 2.9 & 100.0 \\
\hline & 48 & 3 & 11 & 0 & 0 & 4 & 0 & 66 \\
\hline \multirow[t]{2}{*}{$\mathrm{Cmi}$} & 72.7 & 4.5 & 16.7 & 0.0 & 0.0 & 6.1 & 0.0 & 100.0 \\
\hline & 71 & 2 & 14 & 9 & 1 & 16 & 1 & 114 \\
\hline \multirow[t]{2}{*}{ Pub } & 62.3 & 1.8 & 12.3 & 7.9 & 0.9 & 14.0 & 0.9 & 100.0 \\
\hline & 53 & 1 & 9 & 3 & 13 & 19 & 3 & 101 \\
\hline \multirow[t]{2}{*}{$\mathrm{Pba}$} & 52.5 & 1.0 & 8.9 & 3.0 & 12.9 & 18.8 & 3.0 & 100.0 \\
\hline & 133 & 3 & 15 & 4 & 0 & 61 & 5 & 221 \\
\hline \multirow[t]{2}{*}{$\mathrm{Cou}$} & 60.2 & 1.4 & 6.8 & 1.8 & 0.0 & 27.6 & 2.3 & 100.0 \\
\hline & 27 & 0 & 2 & 1 & 1 & 24 & 7 & 62 \\
\hline \multirow[t]{2}{*}{$\mathrm{Coa}$} & 43.5 & 0.0 & 3.2 & 1.6 & 1.6 & 38.7 & 11.3 & 100.0 \\
\hline & 59.4 & 1.7 & 9.4 & 2.8 & 2.5 & 21.4 & 2.8 & 100.0 \\
\hline Totale & 355 & 10 & 56 & 17 & 15 & 128 & 17 & 598 \\
\hline
\end{tabular}

Fonte: Ilfi (1997; 1999; 2001; 2003; 2005) 
Tabella 2 - tavola di mobilità intergenerazionale all'occupazione svolta due anni dopo lo spostamento territoriale: frequenze assolute e percentuali di riga.

\begin{tabular}{|c|c|c|c|c|c|c|c|c|}
\hline \multirow[t]{2}{*}{$\begin{array}{c}\text { Classe sociale di } \\
\text { origine: }\end{array}$} & \multicolumn{7}{|c|}{ Classe sociale due anni dopo la migrazione } & \multirow[b]{2}{*}{ totale } \\
\hline & Non occ. & Borg & $\mathrm{Cmi}$ & Pub & $\mathrm{Pba}$ & Cou & Coa & \\
\hline \multirow{3}{*}{ Bor } & 18 & 2 & 12 & 1 & 0 & 3 & 1 & 37 \\
\hline & 48.6 & 5.4 & 32.4 & 2.7 & 0.0 & 8.1 & 2.7 & 100.0 \\
\hline & 24 & 2 & 24 & 1 & 0 & 15 & 0 & 66 \\
\hline \multirow[t]{2}{*}{$\mathrm{Cmi}$} & 36.4 & 3.0 & 36.4 & 1.5 & 0.0 & 22.7 & 0.0 & 100.0 \\
\hline & 42 & 6 & 23 & 7 & 1 & 37 & 1 & 117 \\
\hline \multirow[t]{2}{*}{ Pub } & 35.9 & 5.1 & 19.7 & 6.0 & 0.9 & 31.6 & 0.9 & 100.0 \\
\hline & 24 & 2 & 12 & 6 & 5 & 59 & 3 & 111 \\
\hline \multirow[t]{2}{*}{$\mathrm{Pba}$} & 21.6 & 1.8 & 10.8 & 5.4 & 4.5 & 53.2 & 2.7 & 100.0 \\
\hline & 78 & 8 & 32 & 6 & 0 & 110 & 2 & 236 \\
\hline \multirow[t]{2}{*}{$\mathrm{Cou}$} & 33.1 & 3.4 & 13.6 & 2.5 & 0.0 & 46.6 & 0.8 & 100.0 \\
\hline & 16 & 0 & 5 & 2 & 0 & 41 & 2 & 66 \\
\hline \multirow[t]{2}{*}{ Coa } & 24.2 & 0.0 & 7.6 & 3.0 & 0.0 & 62.1 & 3.0 & 100.0 \\
\hline & 202 & 20 & 108 & 23 & 6 & 265 & 9 & 633 \\
\hline Totale & 31.9 & 3.2 & 17.1 & 3.6 & 0.9 & 41.9 & 1.4 & 100.0 \\
\hline
\end{tabular}

Fonte: Ilfi $(1997 ; 1999 ; 2001 ; 2003 ; 2005)$ 
Tabella 3 - tavola di mobilità intergenerazionale all'occupazione attuale.

\begin{tabular}{|c|c|c|c|c|c|c|c|}
\hline & \multicolumn{7}{|c|}{ Centro-settentrionali } \\
\hline & Borg & $\mathrm{Cmi}$ & Pub & $\mathrm{Pba}$ & Cou & Coa & Totale \\
\hline Bor & 30,5 & 40,2 & 13,6 & 0,3 & 15,1 & 0,3 & 100,0 \\
\hline $\mathrm{Cmi}$ & 18,8 & 46,1 & 9 & 0,4 & 25,1 & 0,6 & 100,0 \\
\hline Pub & 10,5 & 23,7 & 29,9 & 0,7 & 34,7 & 0,6 & 100,0 \\
\hline $\mathrm{Pba}$ & 2,7 & 13,3 & 14,9 & 17,1 & 46,1 & 6,0 & 100,0 \\
\hline Cou & 5,6 & 25,2 & 13,9 & 0,4 & 54,4 & 0,5 & 100,0 \\
\hline Coa & 1,6 & 6,7 & 14,8 & 4,6 & 61,2 & 11,1 & 100,0 \\
\hline Totale & 8,7 & 25 & 16,7 & 2,8 & 44,9 & 2,0 & 100,0 \\
\hline \multicolumn{2}{|c|}{ Immobili } & 39,7 & 2.0 & & & & \\
\hline \multicolumn{2}{|c|}{ Mobili ascendenti } & 26,7 & 1.3 & & & & \\
\hline \multicolumn{2}{|c|}{ Mobili discendenti } & 19,9 & 1.0 & & & & \\
\hline \multicolumn{2}{|c|}{ Mobili orizzontali } & 13,6 & & & & & \\
\hline & \multicolumn{7}{|c|}{ Meridionali statici } \\
\hline & Borg & $\mathrm{Cmi}$ & Pub & $\mathrm{Pba}$ & Cou & Coa & Totale \\
\hline Bor & 34,3 & 41,8 & 9 & 1,5 & 11,9 & 1,5 & 100,0 \\
\hline $\mathrm{Cmi}$ & 20,8 & 45,5 & 9,6 & 1,1 & 21,9 & 1,1 & 100,0 \\
\hline Pub & 8,5 & 21,3 & 27,9 & 2,2 & 37,4 & 2,7 & 100,0 \\
\hline $\mathrm{Pba}$ & 4,5 & 12,1 & 16,2 & 22,8 & 32,1 & 12,4 & 100,0 \\
\hline $\mathrm{Cou}$ & 5 & 22,3 & 13,8 & 0,9 & 54,2 & 3,9 & 100,0 \\
\hline Coa & 1,2 & 8,1 & 8,1 & 4,9 & 43,9 & 33,7 & 100,0 \\
\hline Totale & 7,5 & 21,6 & 15,5 & 4,9 & 42,1 & 8,4 & 100,0 \\
\hline \multicolumn{2}{|c|}{ Immobili } & 40,5 & & & & & \\
\hline \multicolumn{2}{|c|}{ Mobili ascendenti } & 24,3 & & & & & \\
\hline \multirow{2}{*}{\multicolumn{2}{|c|}{$\begin{array}{l}\text { Mobili discendenti } \\
\text { Mobili orizzontali }\end{array}$}} & 18,5 & & & & & \\
\hline & & 16,7 & & & & & \\
\hline \multicolumn{8}{|c|}{ Meridionali emigrati al Centro-nord } \\
\hline & Borg & $\mathrm{Cmi}$ & Pub & $\mathrm{Pba}$ & Cou & Coa & Totale \\
\hline Bor & 45,5 & 22,7 & 9,1 & 0,0 & 22,7 & 0,0 & 100,0 \\
\hline $\mathrm{Cmi}$ & 14,8 & 51,9 & 18,5 & 0,0 & 14,8 & 0,0 & 100,0 \\
\hline Pub & 16 & 24 & 10 & 0,0 & 50 & 0,0 & 100,0 \\
\hline $\mathrm{Pba}$ & 3,4 & 15,5 & 13,8 & 0,0 & 67,2 & 0,0 & 100,0 \\
\hline Cou & 7,6 & 28,5 & 9 & 0,0 & 54,2 & 0,7 & 100,0 \\
\hline Coa & 2,7 & 8,1 & 16,2 & 0,0 & 67,6 & 5,4 & 100,0 \\
\hline Totale & 10,7 & 24,9 & 11,5 & 0,0 & 52,1 & 0,9 & 100,0 \\
\hline \multicolumn{2}{|c|}{ Immobili } & 32,2 & & & & & \\
\hline \multicolumn{2}{|c|}{ Mobili ascendenti } & 26,3 & & & & & \\
\hline \multicolumn{2}{|c|}{ Mobili discendenti } & 23,7 & & & & & \\
\hline \multicolumn{2}{|c|}{ Mobili orizzontali } & 17,8 & & & & & \\
\hline
\end{tabular}

Fonte: Ilfi (1997; 1999; 2001; 2003; 2005) 
Tabella 4 - Origine geografica e mobilità sociale intergenerazionale per genere. Linear regression panel model with random effects: beta e CIs al 95\% tra parentesi.

\begin{tabular}{lcc}
\hline & Uomini & Donne \\
\hline $\begin{array}{l}\text { Origine geografica [rif:centro- } \\
\text { settentrionali }\end{array}$ & & \\
& & \\
Meridionali statici & $-0.04^{* * *}$ & $0.06^{* * *}$ \\
& $(-0.05--0.04)$ & $(0.06-0.07)$ \\
Immigrati interni & $0.11^{* * *}$ & $-0.01^{* *}$ \\
& $(0.10-0.12)$ & $(-0.02--0.00)$ \\
Tornati & $0.08^{* * *}$ & $-0.28^{* * *}$ \\
& $(0.06-0.09)$ & $(-0.30--0.26)$ \\
\hline Numero di osservazioni & 668,248 & 477,056 \\
\hline Numero di mesi considerati per & 540 & 540 \\
individui & &
\end{tabular}


Tabella 5 - Origine geografica e mobilità sociale intergenerazionale per genere e per istruzione. Linear regression panel model with random effects: beta e CIs al 95\% tra parentesi.

\begin{tabular}{|c|c|c|c|c|}
\hline & \multicolumn{2}{|c|}{ Uomini } & \multicolumn{2}{|c|}{ Donne } \\
\hline & $\begin{array}{c}\text { Licenza } \\
\text { media o meno }\end{array}$ & $\begin{array}{c}\text { Diploma o } \\
\text { laurea }\end{array}$ & $\begin{array}{c}\text { Licenza } \\
\text { media o meno }\end{array}$ & $\begin{array}{c}\text { Diploma o } \\
\text { laurea }\end{array}$ \\
\hline $\begin{array}{l}\text { Origine geografica } \\
\text { [rif:centro- } \\
\text { settentrionali }\end{array}$ & & & & \\
\hline Meridionali statici & $\begin{array}{c}-0.09 * * * \\
(-0.10--0.09)\end{array}$ & $\begin{array}{c}0.02 * * * \\
(0.01-0.03)\end{array}$ & $\begin{array}{c}-0.06 * * * \\
(-0.07--0.05)\end{array}$ & $\begin{array}{c}0.21 * * * \\
(0.20-0.22)\end{array}$ \\
\hline Immigrati interni & $\begin{array}{c}0.16^{* * *} \\
(0.14-0.17)\end{array}$ & $\begin{array}{c}0.03 * * * \\
(0.01-0.05)\end{array}$ & $\begin{array}{c}-0.14 * * * \\
(-0.15--0.12)\end{array}$ & $\begin{array}{c}0.18 * * * \\
(0.16-0.20)\end{array}$ \\
\hline Tornati & $\begin{array}{c}-0.04 * * * \\
(-0.05--0.02)\end{array}$ & $\begin{array}{c}0.25^{* * *} \\
(0.22-0.27)\end{array}$ & $\begin{array}{c}-0.19 * * * \\
(-0.23--0.16)\end{array}$ & $\begin{array}{c}-0.28 * * * \\
(-0.31--0.26)\end{array}$ \\
\hline $\begin{array}{l}\text { Numero di } \\
\text { osservazioni } \\
\text { Numero di mesi } \\
\text { considerati per } \\
\text { individui }\end{array}$ & 430,038 & 238,210 & 272,305 & 204,751 \\
\hline
\end{tabular}

Fonte: Ilfi (1997; 1999; 2001; 2003; 2005) 